\title{
A Study on the DC and Impulse Breakdown Performances of PPLP Insulation in Liquid Nitrogen for DC Applications
}

\author{
W. J. Kim ${ }^{1}$, H. J. Kim², J. W. Cho ${ }^{2}$, S. Hwangbo ${ }^{3}$ and S. H. Kim ${ }^{1, *}$ \\ ${ }^{1}$ Department of Electrical Engineering, Gyeongsang National University and Engineering Research Institute \\ ${ }^{2}$ Superconductivity Research Center, Korea Electrotechnology Research Institute \\ ${ }^{3}$ Department of Electronic and Photonic Engineering, Honam University
}

Received 28 September 2012; accepted 14 November 2012

\begin{abstract}
A high- $\mathrm{T}_{\mathrm{c}}$ superconducting (HTS) DC application has advantages such as the ultimately lower loss, more compact dimensions, and large capacity compared to AC application. In order to optimize the insulation design of a HTS DC machines, it is important to understand the high voltage insulation and materials at cryogenic temperature. Polypropylene laminated paper (PPLP) has been widely used as an insulating material for HTS AC machines. However, the fundamental data under DC voltage have not been revealed satisfactorily until now. In this paper, we will discuss mainly on the breakdown and dielectric characteristics of PPLP in liquid nitrogen $\left(\mathrm{LN}_{2}\right)$. The polarity effects of DC and impulse voltage were studied by using the semi-rod to cylindrical electrode. The volume resistivity of PPLP in $\mathbf{L N}_{2}$ was studied. Also, the space charge distribution at room temperature was studied. However, it is necessary to study this topic at cryogenic temperature in the near future.
\end{abstract}

Keywords: HTS cable, PPLP, Space charge, Dielectric breakdown.

\section{INTRODUCTION}

Recently, HTS machines such as transformer, cable and energy storage magnet are being developed because of their lighter weight, smaller volume, higher efficiency and large capacity than that of the conventional machines. In particular, it has been reported that the HTS cable is the highest in terms of applicability. Therefore, many countries have actively promoted R\&D activities for the development of AC cable [1-3]. Also, the development of high-efficiency HTS DC cables has been promoted to pay attention to the following matters: land long-term, high-capacity power transmission, submarine cable and new \& renewable energy. Because the HTS DC cables are free from AC loss and dielectric loss, DC transmission which has zero loss is possible theoretically.

The HTS cables consist of a conductor, a cooling system, insulating materials and the cryogenic insulation technology. To develop a HTS cable system, it is necessary to develop the cryogenic insulation technology as well as to achieve current capacity and low loss. In particular, cryogenic insulation technology and the material technology of HTS cables must be developed for a long

\footnotetext{
* Corresponding author: shkim@gnu.ac.kr
}

life of cables, high stability and compact system.

For the insulation system of HTS DC cables, a CV cable insulation method and multi-layer insulation method which is the composited insulation system of liquid nitrogen and PPLP are under development. PPLP has been widely used as an insulating material for HTS AC cables. Unlike the conventional AC cables, it is important to understand the characteristics of volume resistivity and space charge distribution. The insulating material with high resistivity is required because of the heat generation problem which is caused by Joule heating in the stationary state current. Also, space charge in the insulating material distorts the electric field distribution in the insulating material and causes breakdown under low DC voltage [4, 5]. For designing electrical insulation of HTS DC machines, it is very important to study the characteristics of DC and impulse breakdown, transient overvoltage superimposed onto DC voltage and polarity reversal DC breakdown. However, many theoretical and experimental studies on the space charge distribution and breakdown of PPLP in $\mathrm{LN}_{2}$ have not revealed satisfactorily until now. Therefore, it has been very difficult to develop HTS DC cables.

We have been studied on the development of HTS AC machines and electrical insulation design as a part of the 21 st century frontier project $[6,7]$. However, it has been almost impossible to study the HTS DC machines. To secure the operating stability, therefore, it is necessary to accumulate a lot of basic data on the materials, insulation as well as dielectric characteristics.

We investigated the breakdown and dielectric characteristics of PPLP at first stage. DC and impulse voltage was applied using the semi-rod to cylindrical electrode. Also, the fundamental characteristics such as method of applied voltage and polarity effect, space charge and resistivity were studied. As a result, PPLP is the excellent insulating material for DC HTS cable as well as for AC HTS cable.

\section{EXPERIMENTAL SETUP AND METHOD}

Fig. 1 shows an electrode system for breakdown. To simulate the HTS cable, stainless-steel cylindrical electrode (diameter: 27, 36, $63 \mathrm{~mm}$ ) and semi-sphere rod 
electrode (diameter: $25 \mathrm{~mm}$, length: $25 \mathrm{~mm}$ ) were used. The $119 \mu \mathrm{m}$-thick PPLP sheet has been used as a sample. The sample was cleaned up with ethylene alcohol and dried in a dryer to remove moisture. Then, it was fixed to the sample holder.

A sample holder was attached to the bottom of the high-voltage bushing on the cryostat. After the commercial liquid nitrogen was inserted, DC and impulse voltage were applied to the electrodes under atmospheric pressure. In the method of applied DC voltage, a short-term breakdown test and a step-based breakdown test. In case of impulse, after applying $70 \%$ of breakdown voltage for initial voltage, breakdown was performed by applying voltage once and three times by $4 \mathrm{kV}$.

A breakdown test was performed once and then the PPLP was replaced with a new one. Breakdown and surface discharge tests were performed 10-20 times at the given conditions.

The space charge distribution under the DC voltage has been measured using the same experimental apparatus [8]. However, it is measured at room temperature because it was non-measurable at cryogenic temperature.

Fig. 2 shows a schematic diagram of measuring system for space charge. A sample is the PPLP with $119 \mu \mathrm{m}$ in thickness and $85 \mathrm{~mm}$ in diameter. For the upper and lower electrodes, a semi-conducting carbon paper with $45 \mathrm{~mm}$ and $30 \mathrm{~mm}$ in diameter was used, respectively.

In addition, there was a $1 \mathrm{~mm}$ gap between the guarded and lower electrodes.

A pulse generator has been used as input pulse to impose displacement on the space charge. Specifically, voltage pulse with $4 \mathrm{kV}$ in size and 5-30 $\mathrm{nm}$ in width has been used. The pressure wave was also transformed into electric signal using the piezoelectric element (PVDF) installed at the bottom of the lower electrode.

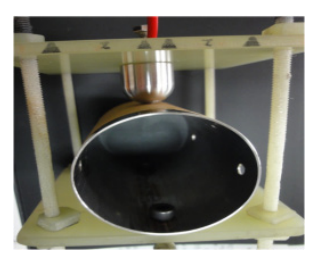

a) Electrode system

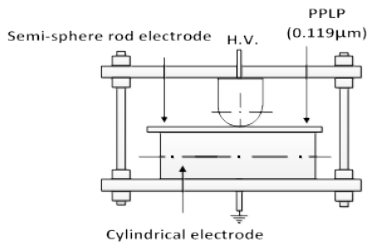

b) Sample holder
Fig. 1. Electrode system for breakdown.

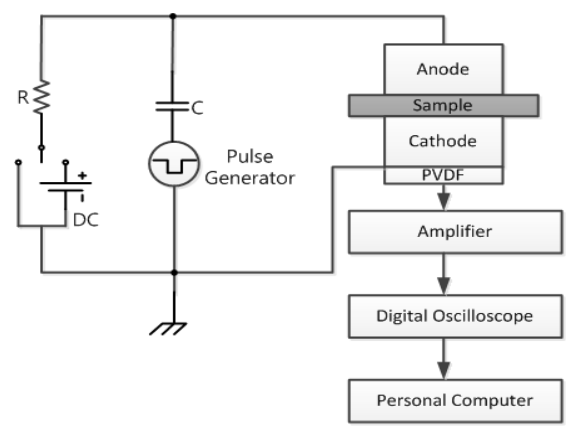

Fig. 2. Schematic diagram of measuring system for space charge.
For the measurement of volume resistivity, the guarded electrode-installed PPLP was impregnated in liquid nitrogen $(77 \mathrm{~K})$, liquid argon $(87 \mathrm{~K})$ and silicon oil $(300 \mathrm{~K})$. Also, the leakage current for one minute was estimated by changing the voltage of 0.5 to $10 \mathrm{kV}$ using the DC high-voltage insulation tester (MEG 10-1, maximum voltage: $10 \mathrm{kV}$, maximum resistance : $500 \mathrm{G} \Omega$ ).

\section{RESULTS AND DISCUSSION}

\subsection{Breakdown Characteristics}

To examine the effect of space charge on breakdown voltage, therefore, a test which differs from the conventional method of AC applied voltage [6] is performed.

Table 1 shows the breakdown by the test methods of applied voltage with DC (+), impulse $(+)$ and AC. The stainless-steel cylindrical electrode of $36 \mathrm{~mm}$ and semi-sphere to rod electrode were used.

In case of DC, a step-based breakdown test which keeps the time of applied voltage to five (DC-2 method) and ten (DC-3 method) minutes was slightly lower than a short-term breakdown method (DC-1 method) in terms of breakdown voltage. However, the impulse breakdown voltage which applied by 1 time (Imp-1 method) is almost same as voltage which applied by 3 times (Imp-2 method).

The DC voltage is applied to the PPLP sheet. Space charge can be formed, which in turn distorted the distribution of electric field inside of the PPLP. As a result, there is a possibility of reducing breakdown voltage by causing high electric field locally. However, small change was observed in terms of breakdown voltage. On the other hand, the change of $\mathrm{AC}$ breakdown voltage is severer than that of DC

At this experimental stage, we cannot make firm conclusions on the relation of breakdown and space charge. There is a possibility that the time of applied DC voltage would be short, degradation would slow down in case of DC and others. As future work, we will conduct the long time breakdown and degradation of DC voltage.

Fig. 3 shows the dependence of DC $(+)$ breakdown voltage on the diameter of cylindrical electrodes. As shown in this figure, the cylindrical electrodes are lower than the plane electrode in terms of DC breakdown voltage. In addition, the DC breakdown voltage is slightly low in the cylindrical electrode with $36 \mathrm{~mm} \Phi$ or less in diameter. However, DC breakdown voltage slightly increases as the diameter of cylindrical electrode increases. Therefore, in the use of small conductor, we must be careful for the insulation design of HTS cable. However, DC breakdown voltage slightly increases as the diameter of cylindrical electrode increases. Therefore, in the use of small conductor, we must be careful for the insulation design of HTS cable.

Fig. 4 shows the dependence of the DC breakdown voltage on the insulation thickness and polarity effect.

When positive voltage and negative voltage are applied to the upper semi-sphere rod electrode, they are called "positive polarity" and "negative polarity", respectively. In 
TABLE I

TEST METHODS OF APPLIED VOLTAGE.

\begin{tabular}{|c|c|c|c|c|}
\hline Voltage & Method & $\begin{array}{c}\text { Initial } \\
\text { Voltage }(\mathrm{kV})\end{array}$ & Step-up & $\begin{array}{c}\text { Breakdown } \\
\text { Voltage }(\mathrm{kV})\end{array}$ \\
\hline \multirow{3}{*}{$\mathrm{DC}(+)$} & DC-1 & 0 & $2 \mathrm{kV} / \mathrm{s}$ & 31.4 \\
\hline & DC-2 & 22 & $\begin{array}{c}2 \mathrm{kV} / \\
5 \mathrm{~min} \text { step }\end{array}$ & 30.8 \\
\hline & DC-3 & 22 & $\begin{array}{c}2 \mathrm{kV} / \\
10 \mathrm{~min} \text { step }\end{array}$ & 30 \\
\hline \multirow{2}{*}{$\underset{(+)}{\operatorname{Imp}}$} & Imp-1 & $\begin{array}{l}22 \times \\
1 \text { time }\end{array}$ & $4 \mathrm{kV}$ & 40 \\
\hline & Imp-2 & $\begin{array}{c}22 \times \\
3 \text { times }\end{array}$ & $\begin{array}{c}4 \mathrm{kV} x \\
\text { 3repetitions }\end{array}$ & 40 \\
\hline
\end{tabular}

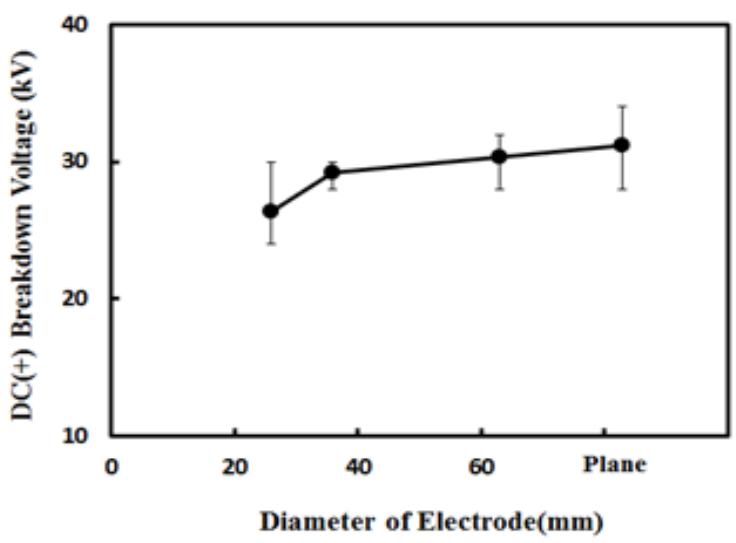

Fig. 3. Dependence of DC breakdown voltage on the diameter of cylindrical electrodes.

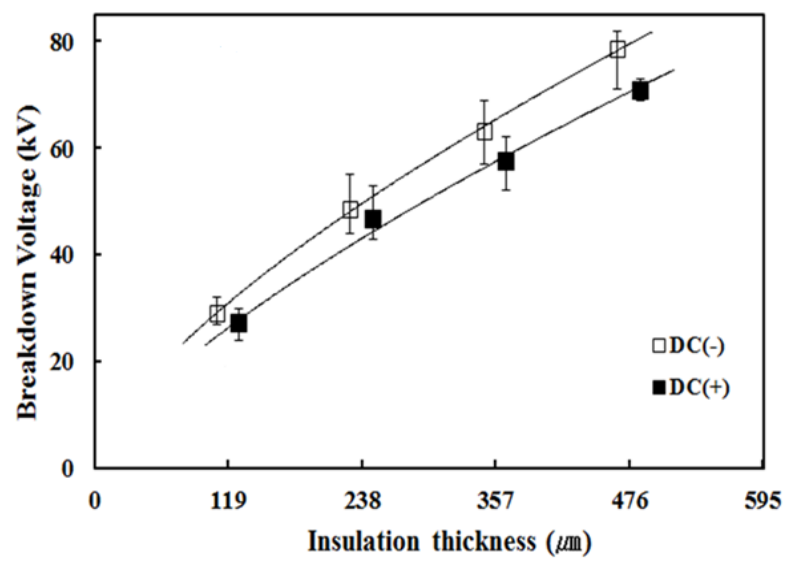

Fig. 4. Dependence of DC breakdown voltage on the insulation thickness and polarity effect.

any cases, breakdown voltage tends to increase as the number of multi-layers increases just as the breakdown voltage of general solid insulating materials. In addition, it has been confirmed that the DC breakdown voltage of negative polarity is proportional to 0.68 square of thickness.

Fig. 5 shows the dependence of insulation thickness and the polarity effect of the impulse breakdown voltage. Just like in DC, negative polarity is slightly higher than the positive polarity in impulse breakdown voltage.

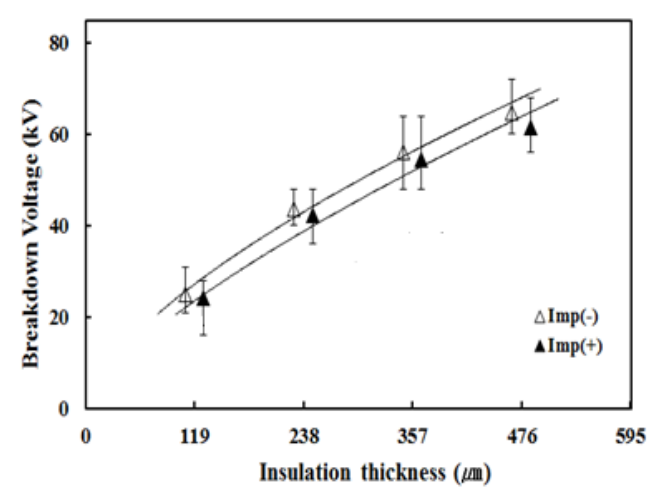

Fig. 5. Dependence of impulse breakdown voltage on the insulation thickness and polarity effect.

However, the impulse breakdown voltage is slightly lower than the DC breakdown voltage. Also, this characteristic has already been reported by Tsuyuki [9].

In any cases, the breakdown voltage tends to exponentially increase as the number of multi-layers increases just as the breakdown voltage of general solid insulating materials. In addition, it has been confirmed that the impulse breakdown voltage is proportional to 0.66 square of the thickness. Consequently, it has been confirmed that PPLP is superior as an insulating material for DC cables as well as for AC cables.

\subsection{Volume Resistivity}

In DC machines, the space charge accumulates in the insulating material. Therefore, it is required for the high volume resistivity.

Fig. 6 shows the temperature dependence of volume resistivity of the PPLP. Temperature was measured at liquid nitrogen $(77 \mathrm{~K})$, liquid argon $(87 \mathrm{~K})$ and silicon oil $(300 \mathrm{~K})$. As shown in this figure, the volume resistivity of PPLP sheet is about $10^{16} \Omega \cdot \mathrm{cm}$ at $\mathrm{LN}_{2}$ temperature. Therefore, the space charge in PPLP seems to be strongly influenced because of its high volume resistivity. As the temperature is increased, the volume resistivity is also decreased. At room temperature, it is about $10^{14} \Omega \cdot \mathrm{cm}$.

\subsection{Space Charge Distribution}

Fig. 7 shows the space charge distribution measured by the pulse electro-acoustic (PEA) method.

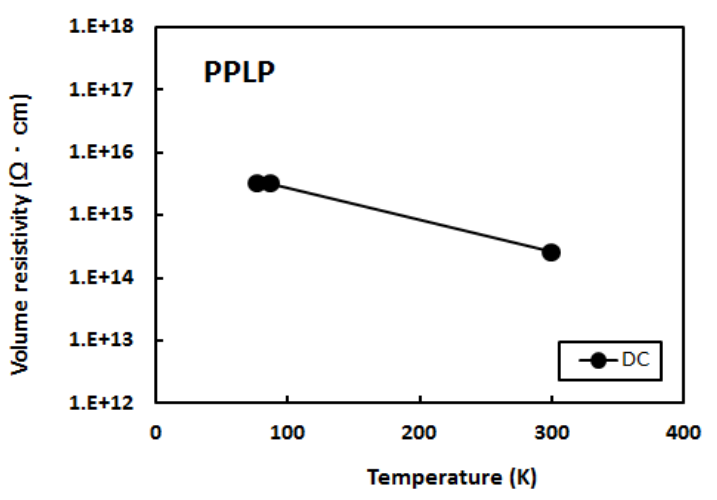

Fig. 6. Temperature dependence of volume resistivity. 


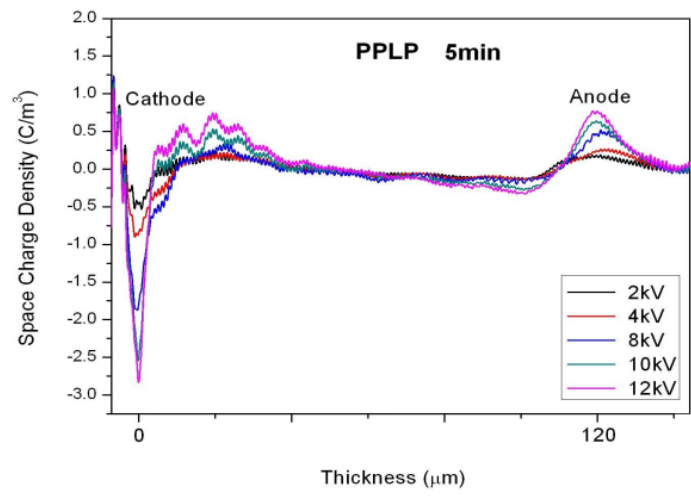

Fig. 7. Space charge distribution under applied voltage.

The charging voltage ranges from 2 to $12 \mathrm{kV}$, charging time of applied voltage is 5 minutes.

As shown in this figure, a positive space charge was formed around the cathode while the negative space charge was observed around anode. In addition, the space charge density gradually increased as the applied voltage increased.

From these experiments, the hetero charge was observed around both electrodes instead of the inside of the PPLP sample, which means that the electron and hole injected from the cathode and anode respectively were partially captured in the trap. It's been known that this kind of accumulation of hetero charge increases the input of carrier in the sample-electrode boundary. In particular, it can be understood that the conduction by electrodes provided in the cathode would play an important role. In addition, the conduction by means of the carrier provided from the electrode is more dominant than the conduction through the carrier created in the sample.

This characteristic at room temperature has already been reported by Nakagawa [10]. However, this characteristic in $\mathrm{LN}_{2}$ has not reported until now. We speculate that the space charge density will decrease at cryogenic temperature, compared to room temperature. This suggests that the space charge of PPLP should be considered in insulation design of HTS DC cable. This will be investigated more in detail in the near future.

\section{CONCLUSION}

As the basic research for insulation design of superconducting DC cable, this study has the fundamental characteristics of PPLP. The following results have been obtained:

(1) As the number of multi-layers of PPLP increases, the DC and impulse breakdown voltages also increase and get saturated slowly. DC is slightly higher than the impulse voltage, and the negative polarity is higher than the positive polarity.

(2) The step-based breakdown method of DC was slightly lower than a short-term breakdown method. AC is more critical than DC in terms of decrease in breakdown voltage because of its discharge degradation over voltage and time.
(3) The cylindrical electrode is lower than the plane electrode in terms of DC breakdown voltage. In addition, the DC breakdown voltage is slightly low in the cylindrical electrode with $36 \mathrm{~mm} \Phi$ or less in diameter. However, as the cylindrical electrode increases in diameter, the DC breakdown voltage slightly increases.

(4) The volume resistivity of PPLP sheets at liquid nitrogen temperature is about $10^{16} \Omega \cdot \mathrm{cm}$. However, it declines as the temperature rises.

(5) The hetero charge is formed on the PPLP sample-electrode boundary. Space charge density gradually increases depending on DC charge voltage.

(6) PPLP has good characteristics of breakdown and volume resistivity for DC cable. However, the space charge of PPLP should be considered in insulation design of HTS DC cable.

\section{ACKNOWLEDGMENT}

This work was supported by a grant from Basic Science Research Program of Korea Electrotechnology Research Institute funded by the Korea Research Council for Industrial Science and Technology (KOCI).

\section{REFERENCES}

[1] S. Mukoyama, M. Yagi, T. Yonemura, T. Nomura, N. Fujiwara, Y. Ichikawa, Y. Aoki, T. Saitoh, N. Amemiya, A. Ishiyama, and N. Hayakawa, "Model Cable Tests for a $275 \mathrm{kV} 3 \mathrm{kA}$ HTS Power Cable", IEEE Trans. Appl. Supercond., vol. 21, no. 3, pp. 976-979, 2011.

[2] J. F. Maguire, J. Yuan, W. Romanosky, F. Schmidt, R. Soika, S. Bratt, F. Durand, C. King, J. McNamara, and T. E. Welsh, "Progress and Status of a $2 \mathrm{G}$ HTS Power Cable to be Installed in the Long Island Power Authority (LIPA) Grid", IEEE Trans. Appl. Supercond., vol. 21, no. 3, pp. 961-966, 2011.

[3] J. W. Cho, J. H. Bae, H. J. Kim, K. D. Sim, S. H. Kim, H. M. Jang, C Y. Lee, D. W. Kim, "Development of a Single-Phase 30m HTS Power Cable", Cryogenics, vol. 46, pp. 333-337, 2006.

[4] Y. Murakami, S. Mitsumoto, M. Fukuma, N. Hozumi, and M. Nagao, "Space Charge Formation and Breakdown in Polyethylene Influenced by the Interface with Semiconducting Electrodes", $T$. IEE Japan, vol. 120-A, pp. 930-935, 2000.

[5] A. Bradwell, "Conduction in Polyethylene with Strong Electric Fields and the Effect of Pressing on the Electric Strength", Processing of I.E.E., vol. 18, pp. 247-254, 1971.

[6] D. S. Kwag, H. G. Cheon, J. H. Choi, H. J. Kim, J. W. Choi, and S.H. Kim, "Research on the Insulation Design of a $154 \mathrm{kV}$ Class HTS Power Cable and Termination", IEEE Trans. Appl. Supercond., vol. 17, no. 2, pp. 1938-1942, 2007

[7] J. H. Choi, J. W. Choi, S. M. Baek, and S. H. Kim, "The Insulation Design of $154 \mathrm{kV}$ HTS Transformer and on Load Tap Changers", IEEE Trans. Appl. Supercond., vol. 19, no. 3, pp. 1972-1975, 2009.

[8] S. Hwangbo, D. S. Shin, D. H. Yun, and M. K. Han, "Effects of Space Charge on the Electrical Conduction Characteristics in a LDPE with Semi conductive Electrodes", Trans. KIEE Korea, vol. 46, no. 8, pp. 1240-1248, 1997

[9] K. Tsuyuki, S. Washida, O. Tanda, T. Masuda, K. Kato, T. Nakajima, and S. Mukoyama, "Study on the Electrical Insulation of High-Tc Superconducting DC Power Cable", Cryogenic Engineering of Japan, vol. 35, no. 7, pp.350-356, 2000.

[10] T. Nakagawa, "Measurement of Space Charge Accumulation in PPLP", Proceedings of 13 th International Conference on Dielectric Liquids (ICDL ’99), Japan, pp.533-536, July 20-25, 1999. 